\title{
DNA sensing unchained
}

Cell Research (2013) 23:585-587. doi:10.1038/cr.2013.28; published online 19 February 2013

In two recent reports in Science, James Chen and colleagues provide compelling evidence that detection of cytosolic DNA triggers the production of a novel second messenger, cyclic GMP-AMP (cGAMP), which in turn activates a signaling pathway that induces type I interferons (IFNs) in a STING-dependent manner. They further unravel a key role for a so far uncharacterized murine protein E330016A19 (human homolog: C6ORF150), now termed cGAMP synthetase (cGAS), to act as the DNA sensor that generates cGAMP.

Innate immune detection of nucleic acids is a central component of the host response to infection with a wide range of pathogens. Pattern recognition receptors (PRRs) of distinct subcellular localization and differential cellular distribution provide sensing platforms for recognition of foreign DNA and RNA, respectively. Accumulation of cytosolic DNA activates a host response, which is characterized by AIM2-dependent secretion of IL- $1 \beta$ and STING-mediated activation of the TBK1-IRF3 axis to induce antiviral gene expression [1]. AIM2 directly interacts with dsDNA, thereby leading to the assembly of an inflammasome complex that culminates in caspase- 1 activation and subsequent maturation of pro-IL-1 $\beta$. STING, however, lacks an obvious DNA-binding domain and thus it was reasoned that an additional factor(s) would act upstream of STING linking DNA binding to activation of downstream signaling. Up to now, a number of candidate DNA receptors have been proposed, including DAI, IFI16, LRRFIP1, Ku70, DDX41 and DNA-PK, yet except for DNA-PK, their in vivo relevance remains uncertain [1,
2]. Intriguingly, besides its requirement for cytosolic DNA sensing, STING was also shown to mediate the intracellular response towards bacterial-derived cyclic di-nucleotides by directly interacting with cyclic di-GMP/AMP [3]. This latter finding was further confirmed by several independent groups solving the crystal structure of cyclic di-GMP bound to STING. Given its unequivocal function as a receptor for cyclic di-nucleotides, the concept of STING serving as an adaptor molecule at the same time was challenging. As such, the novel work from the Chen lab [4, 5], introducing cGAS and its product cGAMP in cytosolic DNA recognition, marks an important breakthrough in innate immunity, reconciling the above-mentioned, seemingly disparate functions of STING.

To study cellular events upstream of STING following DNA challenge, $\mathrm{Wu}$ et al. [5] first established an in vitro complementation assay. Cellular extracts from STING-silenced, DNAstimulated L929 cells were transferred to permeabilized THP1 cells, wherein activation of IRF3 dimerization was monitored. Using this approach the investigators demonstrated that a transferable, IRF3 activating factor was generated upon DNA stimulation and that multiple DNA species were able to induce this factor. Surprisingly, this transferable factor was neither a protein nor a nucleic acid, yet STING was required in the recipient cells to respond to this factor. Consistent with the cell type specificity for DNA-induced signaling, only cells that are known to respond to cytosolic DNA were able to induce this factor. Performing mass spectrometry of STING-bound molecules led to the identification of cGAMP as the critical factor. Indeed, chemically synthesized cGAMP turned out to be a potent inducer of IRF3 phosphorylation and subsequent type I IFN induction. In addition, the critical role for STING in cGAMP sensing was proven in gainand loss-of-function studies in cellulo and direct binding of cGAMP to STING was demonstrated in vitro. With regards to the physiological relevance of this finding, the authors further showed that both HSV-1 and vaccinia virus (VACV) induce cGAMP in human and murine cells. Altogether, these studies nicely demonstrated that certain cell types synthesize cGAMP upon cytosolic DNA delivery to activate STING.

But what protein is responsible for cGAMP synthesis in eukaryotic cells? Chen and colleagues addressed this question as well, with an intriguing result. Cellular extracts from DNAresponsive L929 cells were collected and tested for their ability to produce cGAMP. Different purification routes yielded three proteins that copurified in active fractions. Among them, E330016A19 turned out to be the most promising candidate, given the presence of a conserved domain found in nucleotidyltransferases. In addition, the human homolog of E330016A19, C6ORF150, was recently characterized as an interferon-inducible gene, whose overexpression inhibited replication of several distinct classes of viruses [6]. In fact, overexpression of murine cGAS $($ cGAMP synthetase $=$ E330016A19) in the presence of STING led to potent type I IFN induction. Moreover, overexpression of cGAS in cells or incubation of cGAS with nucleotides and DNA in vitro was sufficient for the genera- 


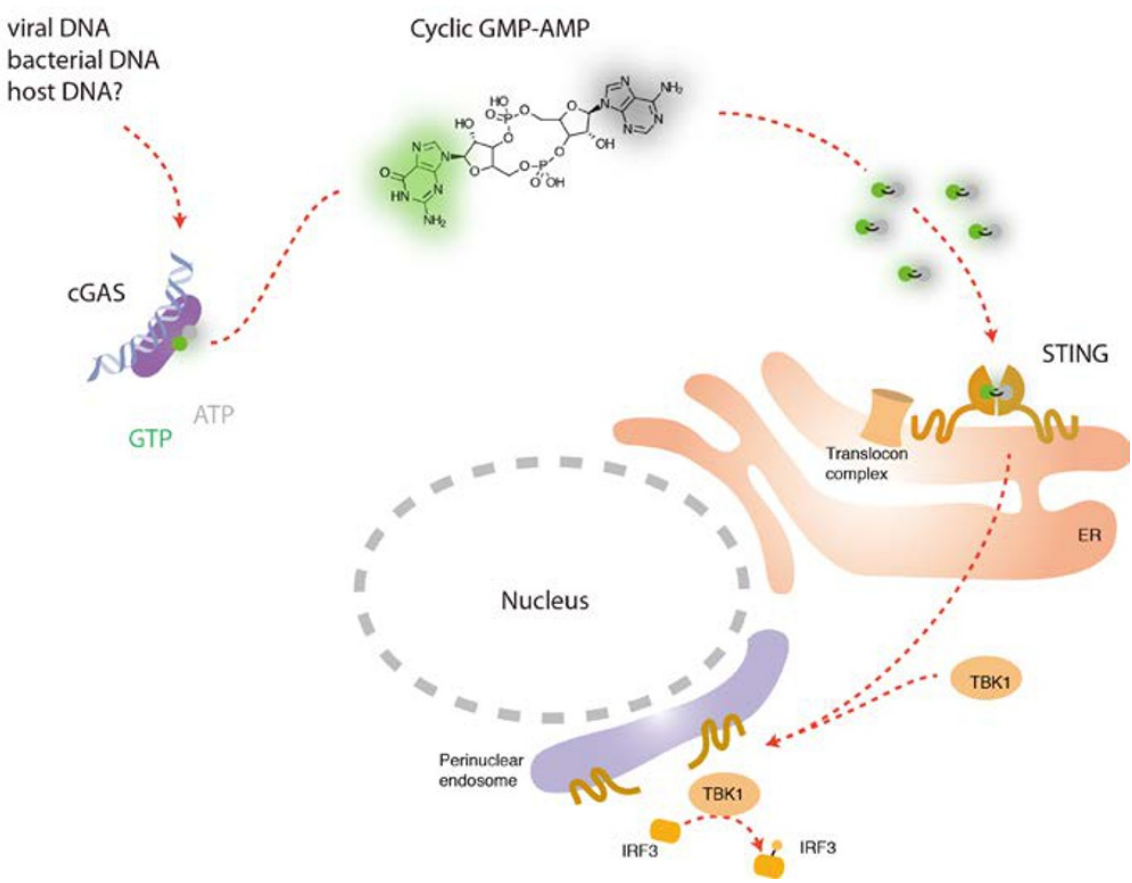

Figure 1 During infection, microbial DNA gains access to the cell cytoplasm and binds to cGAS. This interaction activates cGAS to synthesize the second messenger cGAMP, which in turn is recognized by ER-localized STING. This event leads to translocation of STING to perinuclear endosomes and recruitment of the kinase TBK1. Finally, this series of events induces the transcriptional upregulation of IFN- $\beta$ and additional interferon-stimulated genes. It remains to be determined whether this pathway also accounts for accidental recognition of self-DNA in autoimmune and autoinflammatory conditions.

tion of cGAMP, whereas distinct point mutations within the putative catalytic domain of cGAS abrogated the activity of cGAS. The authors found that knocking down cGAS in either human or mouse cells compromised the activity of cells to respond to DNA or DNA viruses like HSV-1 or VACV, confirming a prominent role of cGAS in DNA sensing. Further studies revealed that cGAS was able to bind DNA directly and that this activity was dependent on the Nterminal portion of the protein. Finally, the investigation of cGAS localization indicated that cGAS was predominantly localized in the cytoplasm, and that it could form distinctive aggregates colocalizing with transfected DNA.

Altogether, these two studies provide compelling evidence that cGAS directly senses the presence of cytosolic DNA to produce cGAMP, which in turn activates
STING to induce antiviral immunity (Figure 1).

After all, should this be the end of our search for the cytosolic DNA sensor? It seems so. At least the concept provided by Chen and colleagues leaves only little room for additional cytosolic DNA sensing mechanisms that could complement the cGAS system. Of course, a knockout cell line or animal will be required to provide the final proof of its non-redundant function in DNA sensing. Nevertheless, it is possible that additional factors contribute to or modulate the cGAS-STING axis in type I IFN induction, which still leaves some space for the previously reported STING-dependent DNA sensing mechanisms, such as DDX41 or IFI16 $[7,8]$. Yet again, knockout studies are necessary to solve the contribution of these factors.
It is interesting to note that cGAS-dependent DNA sensing operates through the detour of a second messenger signaling system as opposed to a "conventional" protein-protein interaction-based signaling cascade, as it is often found in PRR signaling. Second messenger systems are known to greatly amplify the strength of a signal, furthermore, they usually induce pleiotropic effects, as many downstream targets are affected. Moreover, the same second messenger is often employed by different upstream receptors, even though specificity can be achieved by spatiotemporal regulation. cGAMP, however, appears to be a unique and highly specific second messenger, which poses the question of its origin in evolution and its possible function prior to serving as a cell intrinsic STING ligand within the innate immune system. In this context, it is tempting to speculate that cGAMP exerts additional effects, independent of its activity as a STING ligand.

Finally it will be interesting to study the role of cGAS in antimicrobial immunity and sterile inflammation in vivo. Conditions, in which cytosolic DNA sensing has already been linked to STING activation, will be top on the list. As such, disease models with specific deficiencies in DNA degrading enzymes (e.g., TREX1 and DNase II alpha) and a documented role of STING-driven sterile inflammation will be of great interest $[9,10]$. Assuming that cGAS is required for DNA-dependent inflammation under these conditions, targeting cGAS synthetase activity or the binding of its product cGAMP to STING using a small molecule inhibitor could open new avenues in anti-inflammatory therapy. In this context, determining the crystal structure of cGAS, especially its catalytic domain, will be of great value.

\section{Andrea Ablasser ${ }^{1}$, Veit Hornung ${ }^{1}$ \\ 'Institute for Clinical Chemistry and Pharma- cology, Unit for Clinical Biochemistry, Univer- sity Hospital, University of Bonn, Germany Correspondence: Veit Hornung}


E-mail: veit.hornung@uni-bonn.de

\section{References}

1 Cavlar T, Ablasser A, Hornung V. Immunol Cell Biol 2012; 90:474-482.

2 Ferguson BJ, Mansur DS, Peters NE, et al. elife 2012; 1:e0047.
3 Burdette DL, Monroe KM, Sotelo-Troha K, et al. Nature 2011; 478:515-518.

4 Sun L, Wu J, Du F, et al. Science 2013; 339:786-791.

5 Wu J, Sun L, Chen X, et al. Science 2013; 339:826-830.

6 Schoggins JW, Wilson SJ, Panis M, et al. Nature 2011; 472:481-485.
7 Unterholzner L, Keating SE, Baran M, et al. Nat Immunol 2010; 11:997-1004.

8 Zhang Z, Yuan B, Bao M, et al. Nat Immunol 2011; 12:959-965.

9 Gall A, Treuting P, Elkon KB, et al. Immunity 2012; 36:120-131.

10 Ahn J, Gutman D, Saijo S, et al. Proc Natl Acad Sci USA 2012; 109:19386-19391. 